\title{
Syndromes of Related Development of Higher Mental Functions
}

\author{
Janna M. Glozman ${ }^{1, *}$ and Sofia M. Konina ${ }^{2}$ \\ ${ }^{1}$ Lomonosov Moscow State University, Moscow, Russia \\ ${ }^{2}$ Luria Research Centre of Developmental Neuropsychology, Moscow, Russia
}

\begin{abstract}
The paper is aimed to develop Luria's theory of the syndrome analysis to developmental neuropsychology through qualitative and quantitative studies of higher mental functions underdevelopment. A neuropsychological study of the development of higher mental functions in preschool children is considered primordial to prevent a risk of further learning problems. A comprehensive program of neuropsychological remediation effectively helps to overcome revealed difficulties and is a means for prevention of learning problems at school.
\end{abstract}

Keywords: A.R. Luria, developmental neuropsychology, underdevelopment of higher mental functions, qualitative assessment, quantitative assessment.

\section{THEORETICAL INTRODUCTION}

Developmental neuropsychology is a branch of neuropsychology, studying the interaction between a child's social functioning (behaviour and education) with development of his mental functions and personality on one hand and ontogenetic brain development (individual development from this child's birth) on the other in normal and pathological conditions as well as possibilities of using this knowledge for child education and remediation.

We witness now a considerable increase in number of studies and publications that deal with children normal and abnormal development and describe different methods of remediating education. The reason for this is first a general democratization of world society, main manifestation of which is a greater attention to children population. Second, psychologists and educators all over the world are concerned about the growing number of children without evident medical problems who nevertheless show health problems or learning disability and maladjustment at school and in family settings.

The mental and social functioning of such a child does not correspond to his psychophysiological abilities and to his needs as well as to the demands of his social environment.

Luria's neuropsychological assessment permits to differentiate learning and behaviour problems due to individual features of mental functioning from maladjustment caused by wrong educative procedures or negative aspects of the child's personality.

The theoretical basis of developmental neuropsychology as well as of general neuropsychology comprises three principles, proposed by L.S. Vygotsky [1] and A.R. Luria [2]:

*Address correspondence to this author at the Lomonosov Moscow State University, Moscow, Russia; E-mail: Glozman@mail.ru the principle of social genesis of higher mental functions (HMF); the principle of system (syndrome) organization of HMF; the principle of dynamic organization and localization of HMF.

To understand the theory of system or syndrome structure of HMF in humans the following factors are the most important: a variability of interfunctional relations and connections; a formation of complex dynamic systems, integrating many elementary functions; generalized reflection of environment by conscience and mediated mental functioning.

Are the functional components proposed by Luria for syndrome analysis and rehabilitation of adults useful for neuropsychological assessment and remediation of children? Upon T.V. Akhutina, "the functional divisions in components («factors») in children must be a subject of a special and long study. The starting point can be a comparison of success in operations related to the same or different functional components, using as an initial working hypothesis the functional division in adults (considering that it is sufficiently stable in adults and the developing functional systems of children are supposed to reach the same" [3, p. 203]. Another important reason for use of factor analysis in children is the greater efficiency of remediation, based upon data of Lurian neuropsychological assessment revealing disturbed and preserved factors of mental functioning to compare with general development of mental functions.

Luria's principle of dynamic ontogenic localization of HMF means variable level and type of different included brain structures and of their functional integration while realizing the same cognitive activity by children of different age. This Luria's principle proves the necessity to assess child's higher mental functions as early as possible, starting from the preschool age. 
2. THE DEVELOPMENT OF HIGHER MENTAL FUNCTIONS IN PRESCHOOL CHILDREN IS VERY INTENSE

Language development goes in several directions: an improvement in communicating with other people is the basis for the restructuring of mental processes, a tool for reasoning. The preschool child's speech becomes a means of planning and regulation of own behavior [4]. The development of perception at this age, in fact, is a development of methods and means of orientation in space and what about. Studies by L.A. Wenger [5] proved assimilation in preschool years of sensory standards dynamic representations of varieties of objects' individual properties (color, shape, size) and a correlation of relevant objects with these standards. During preschool age with the emergence of more complex activities in children play the attention concentration and stability increase. For example, while younger preschoolers can participate in the same game for about 30-40 minutes, in five to six years the duration of the game increases to one hour and a half [6]. Child's memory is mostly involuntary, the child often does not set a conscious goal to remember something. As was shown by P. Zinchenko [7], the play activity suppose an involuntary memory corresponding to the purpose of the game. At the end of preschool age a voluntary memory begins to develop. At preschool age such operations of reasoning as classification, generalization, establishing causal relationships are in the process of formation [4].

\section{IN A NEUROPSYCHOLOGICAL ASSESSMENT OF CHILDREN}

Especially in the pre-school age, it is necessary to pay special attention to the used diagnostic material. It should be accessible, familiar and attractive (can attract attention). Test stimulus must be carried out mainly as a clear colored image. Objects should be depicted as clearly as possible, and should not include abstract details. Also, one must consider the limitations of perception and attention in preschoolers. In conducting a neuropsychological assessment of a preschooler the specialist needs to give the child a possibility to switch to different types of activities, it is necessary to select the most informative and time-saving methods and techniques. Game-like form is the most suitable type of test (given the main activity in this age group) [8]. These principles of neuropsychological assessment were followed in our experimental study of neuropsychological syndromes in the preschool age.

\section{SUBJECTS AND METHODS}

The research involved 30 children assessed in our Research Centre of Developmental neuropsychology in the period of 2011-2013 because of different complaints, mainly due to the difficulty of mastering the material needed to prepare for school. During the assessment of each child his parents filled up a questionnaire on early development. Neuropsychological assessment included quantitative and qualitative analysis aimed to reveal the strengths and weaknesses of the child's mental development needed to develop strategies and methods of remediation for this child.

All children participated in our study had no evident organic lesion of the central nervous system; these children were trained in a public kindergarten, all lived in good conditions. Among the subjects there were 20 boys and 10 girls, 16 of them were right-handed, 10 left-handed and 4 ambidextrous. Fifteen children in our sampling underwent a neuropsychological follow-up after the course of neuropsychological remediation consisting of complex motor, cognitive exercises and special breathing exercises aimed to overcome the weaknesses of mental activity. Survey of children was performed using the material of Luria neuropsychological battery, adapted in our center for children with qualitative and quantitative analysis of results [8]. The specificity of the rating system, we proposed, based on the qualitative analysis [9] consists in two related but distinct procedures and possibilities of application:

1. A list of possible constraints for each test proposed with consecutive qualification of performance in the analyzed mental sphere in accordance with this list. In result, first, the child's HMF can be expressed as a constellation of +/- (present/absent) symptoms (with a possibility of quantification of the percent of present symptoms from all possible). Secondly, a typical neuropsychological pattern for the assessed groups of children can be described. Thirdly, it is possible to group qualitative parameters (symptoms) on the basis of their common relation to the functioning of the brain structure or the block in order to compare the degree of immaturity of different areas of the brain of the assessed child or children from the age group and to determine the sustainability of symptoms during the follow-up, fourthly, the positive or negative dynamics in state in follow-up can be assessed through disappearance/appearance of certain symptoms, changing neuropsychological pattern.

2. Scoring the performance of each test on the 6-point scale based on identified problems (errors), their primary or secondary nature, their degree of severity compared with the normative data of the relevant age and cultural group, as well as the possibilities and conditions of error correction (necessary way of presenting the material, the degree of child stimulation and the assistance of the examiner) when a neuropsychological assessment has a form of a dialogue. Scoring also allows determining the total score of deficiency for each mental function and the summarized score for the child in order to objectively assess the degree of immaturity (not typical development, deficiency of mental functioning), the dynamics of the child state during a follow-up in the process of remediation.

\section{THE RESULTS OF THE RESEARCH}

Let us compare first the development of different mental functions traditionally assessed in Lurian neuropsychological assessment.

An analysis of scores for mistakes in different tests (Fig. 1) shows that most often the cause of child's problems are low neurodynamic features of mental activity (average 


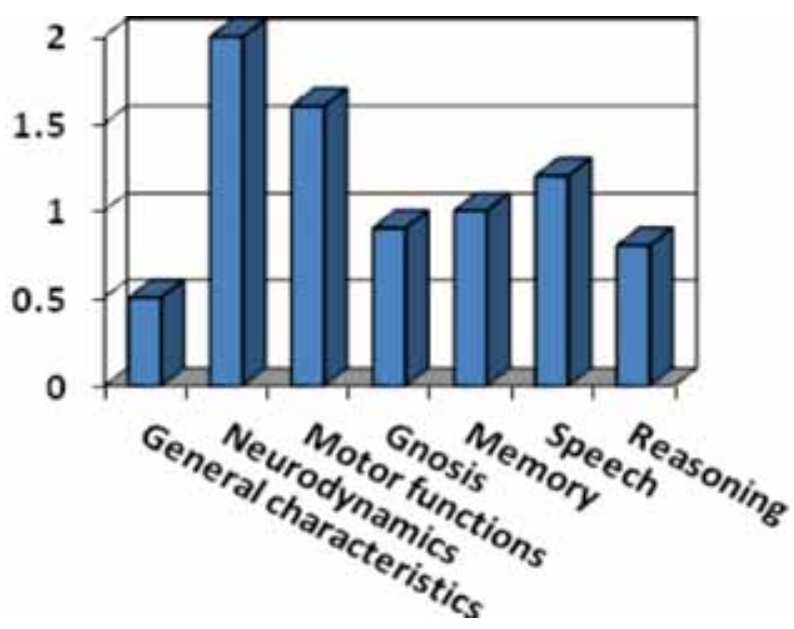

Fig. (1). The degree of severity of underdevelopment in different mental spheres (scores).

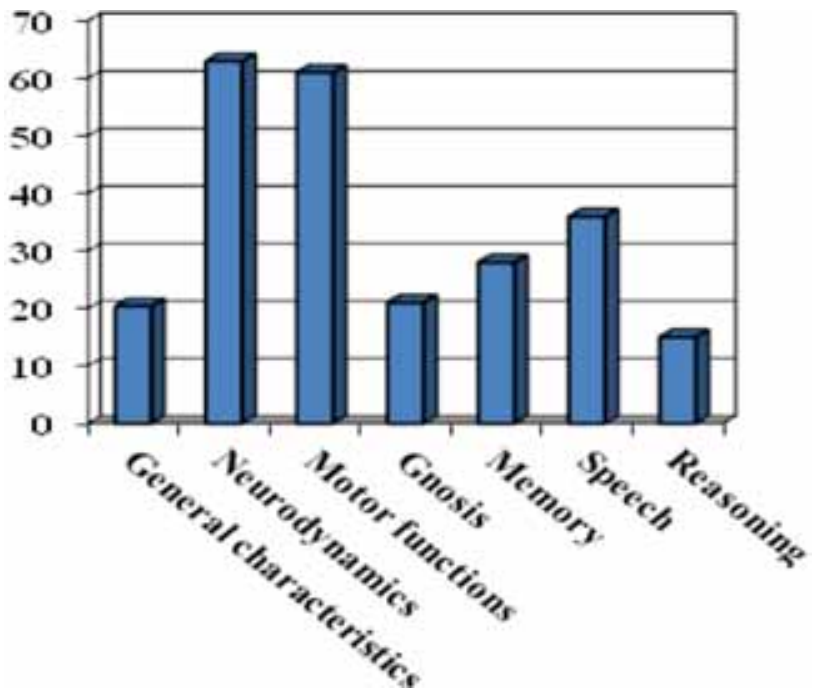

Fig. (2). Number of underdevelopment symptoms in different mental spheres (\%).

score is 2 from maximal score 3 ). It coincides with the complaints of parents that their children are smart, but inconsiderate, often hyperactive, but tire quickly in training tasks etc. The analysis of neurodynamics was conducted through both a proofreading test and evaluation of the child behavior throughout the process of assessment. On the second place, there are difficulties in motor functions: reciprocal coordination of hand movements, oral praxis, digital praxis, the spatial organization of graphic activity, dynamic movements and verbal regulation of movements when reproducing rhythms and implementation of conditioned reactions. Average score for motor functions is 1.6. Next place belongs to the underdevelopment of speech: prosodic, lexical and grammatical components of spontaneous speech, phonemic discrimination, naming of low-frequency words, understanding of logical-grammatical constructions, use of verbal sequences. Mnestic function was investigated by tests on acoustic, visual and motor memory. Errors in these samples may indicate the difficulty of remembering the material required to prepare for school and for successful everyday living of the child. Gnostic functions were assessed using tests for visual, spatial, tactile, color and acoustic gnosis. Intellectual functions were assessed with tests for generalization (to find a common category for images), describing pictured stories and understanding of the meaning of an oral story. Under the general characteristics of the child, we mean such features as critical attitude to own behavior, general knowledge and general behavior during assessment. The development of two last mental functions was better than of the previous ones.

A qualitative analysis of symptoms of underdevelopment (Fig. 2) suggests that first in the preschool years the weakness of the first functional unit of the brain - unit of activation (upon Luria [2]) is revealed. Energy deficit provokes that the child does not have the resources to perform many operations depending mainly from the second unit of the brain (unit of processing and storage of 


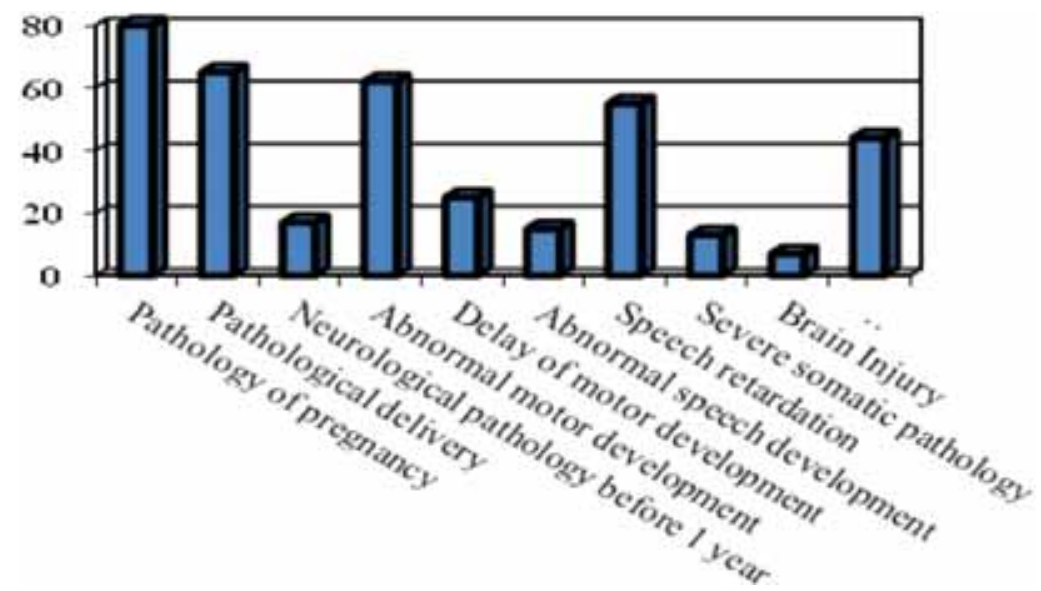

Fig. (3). Relationship of the underdevelopment of mental functions with the features of early development (\%).

information), even when the last was sufficiently mature for this operation. These findings are supported by the described above quantitative results in preschool children. Also, quite often (in 61\%) the third unit of the brain - unit of regulation, monitoring and programming of activity was deficient. The frontal lobes according to A.R. Luria are characterized by a great complexity of structure and by bilateral relationships with cortical and subcortical structures. According to D.B. Elkonin [10] an attitude to a rule as to a condition is a component of a child's readiness for school. Paramount is the verbal regulation of behavior when external and internal verbal instructions regulate child's selectivity of action. It can be formed only in play activities. Underdevelopment of this brain unit coincides with the complaints of parents that their child has a low self-control, has problems to follow the rules, and therefore he violates the rules of behavior in the family and kindergarten. Our study revealed great difficulties in the test of conditioned reactions in which the echopraxic reactions appeared in conflict conditions, as well as the deficient dynamic movements - in situations of switching from one element to another one: disturbances of a smooth transition from one movement to another or from one graphic pattern to another. Also great difficulties arose in the test evaluating the interhemispheric interaction (reciprocal coordination of hands).

Less than these two units described suffered the formation of the second functional unit of the brain - unit of storage and processing of information. Functioning of this unit provides the modality-specific processes, as well as complex integrative forms of information processing necessary for the maturity of higher mental functions.

Let us analyze further the mechanisms of the revealed underdevelopment through correlations of these defects with data of the early development questionnaire (Fig. 3), filled up by the child's parents.

It may be seen that the majority of children had a pathological situation during pregnancy and/or delivery: a threat of termination, infection or other health problems in future mothers, as well as such negative conditions as mechanical squeezing of fetus, cesarean section, etc. Also, quite often (in $62 \%$ of cases) some anomalies occur in the early motor development - a lacking or irregularity in motor development during the first year of the life. The third high incidence regards the delayed speech development. $36 \%$ of children had neurological diagnoses, most commonly ADHD and MMD (minimal mental dysfunction).

An analysis of relationship between qualitative and quantitative assessment scores and the data of early development questionnaires using Statistica 10.0 and Spearman correlation coefficient reveals statistically significant values (> 75). Pathology of pregnancy and delivery correlated in our study with a decrease of neurodynamics (underdevelopment of the first unit of the brain), with abnormal and delayed motor functions and executive functions (the third unit of the brain).

6. Most of the assessed children followed neuropsychological remediation and the neuropsychological follow-up after remediation. Statistical analysis of these data was performed through the Wilcoxon nonparametric test of significance (significant values > 0.005). We see (Fig. 4) that the most pronounced amelioration can be observed in the field of neurodynamics, as well as in the motor functions. Memory function, gnostic function also improved after remediation. Speech and reasoning dynamics is less pronounced.

Our study confirms that a course of a comprehensive neuropsychological remediation consisting of cognitive, motor, and breathing exercises, can in most cases about completely correct neurodynamic, executive and motor difficulties [11]. A consecutive focus of the remediation work on the cognitive functions leads to good results in the functioning of the second unit of the brain.

To confirm that the observed dynamics is associated with remediation and not with retesting, we compared two protocols of 6 years old children, with the same features of early development, the same sex, both right-handers, both children at the time of the assessment studied in the preparation to school group of the kindergarten. One of them followed a course of neuropsychological remediation during 


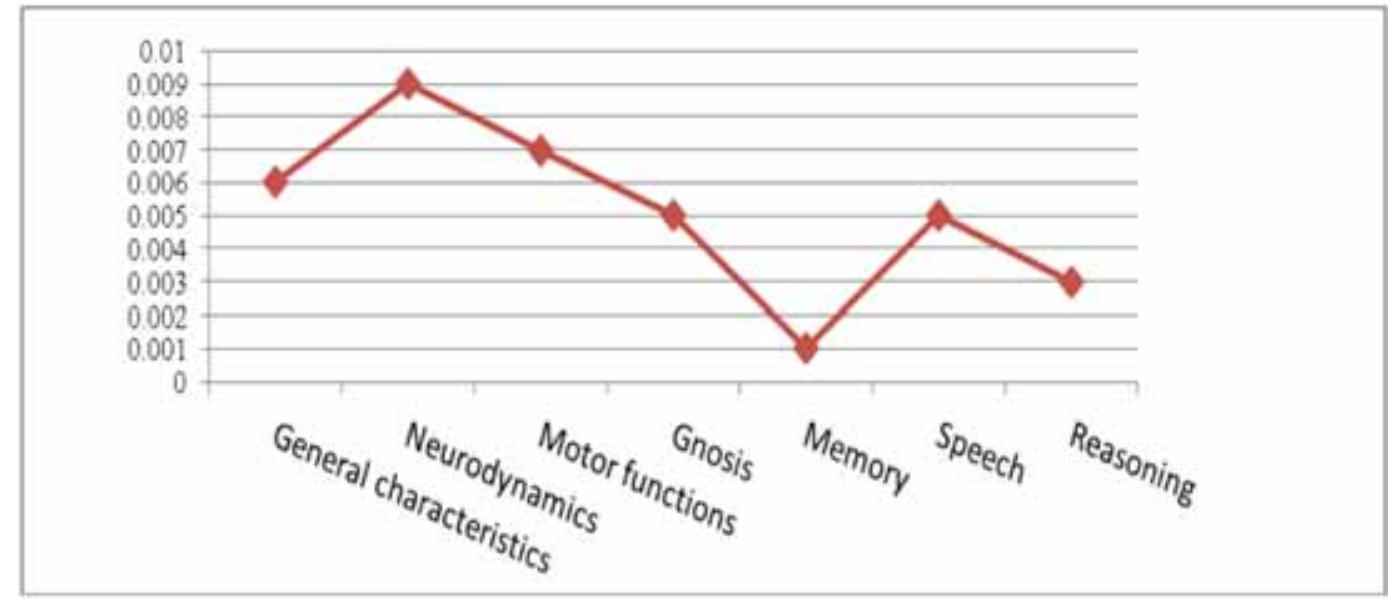

Fig. (4). The role of early neuropsychological remediation in overcoming defects of higher mental functions.
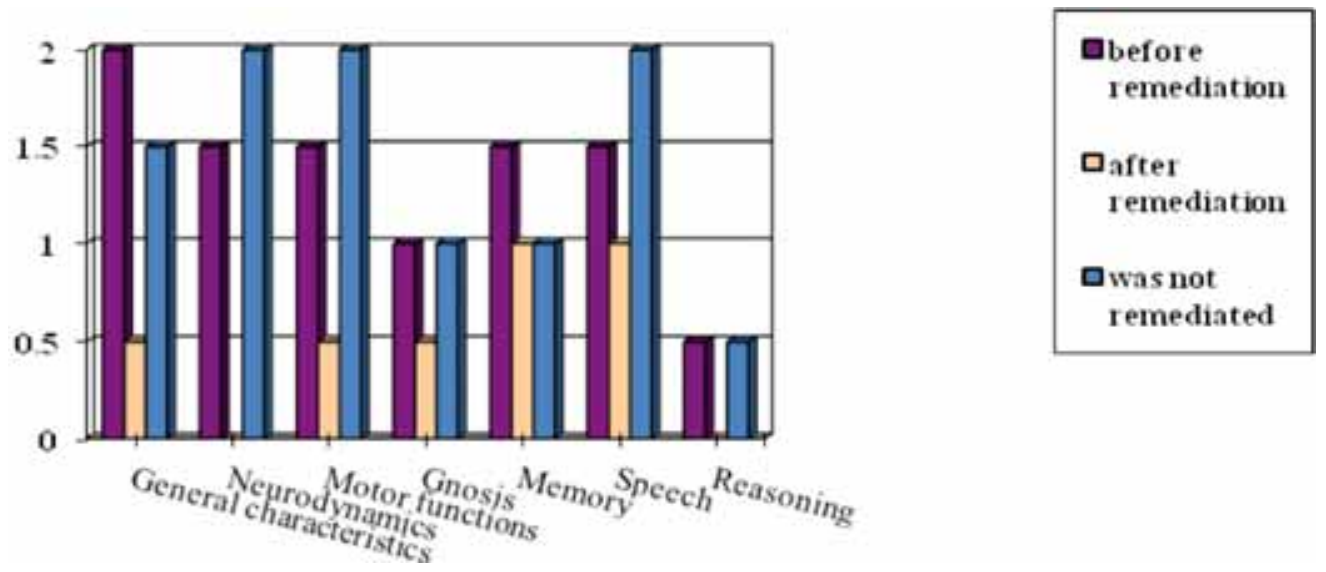

Fig. (5). Comparison of children who followed and did not followed the neuropsychological remediation (scores).

three months; the other did not. The neuropsychological follow-up was effectuated six months after the initial assessment (Fig. 5).

We can see that the neurodynamic scores deteriorated in the child who did not follow the neuropsychological remediation while in his peer they normalized, motor functions became worse in the first boy and much better in the second one. Gnostic, verbal and intellectual functions remain essentially unchanged; memory scores became better in both children. This proves that the neuropsychological remediation in the preschool age may be a prevention of difficulties in school.

The research enables us to draw the following conclusions:

1 The leading syndrome of underdeveloped higher mental functions in the preschool age is attention deficit disorder and difficulties of voluntary regulation and control of own activities.

2 A comprehensive program of neuropsychological remediation corresponding to the current level of child development and meeting modern requirements for the organization of classes, effectively helps to overcome revealed difficulties.
3 Neuropsychological remediation at preschool age can be considered a means for prevention of learning problems at school.

\section{CONFLICT OF INTEREST}

The authors confirm that this article content has no conflicts of interest.

\section{ACKNOWLEDGEMENTS}

The study is partially funded by RHSF project \#13-0600570 and by RSF project \#14-18- 03253.

\section{REFERENCES}

[1] Vygotsky L. Educational Psychology. St. Lucie Press: Florida, 1992.

[2] Luria AR. The Working Brain. An Introduction to Neuropsychology. Penguin Books: London 1973.

[3] Akhutina TV. Neuropsychology of children individual differences as a basis of using neuropsychological methods at school. In: Khomskaya ED, Akhutina TV, Eds. Proceedings of the First International Luria Memorial Conference. Moscow: Russian Psychological Association Press 1998; pp. 201 - 8.

[4] Yermolayeva MV. Psychology and developing remedial work with preschoolers. Moscow: SAG 1998. 
[5] Wenger LA. Formation of cognitive abilities in preschool age. In: Readings in child psychology. Moscow: Psychological Institute Press 1996; pp. 133-46.

[6] Baskakova IL. Attention in preschooler, methods of study and development. In: Attention study in school children. Moscow MODEK: Voronezh: SAG 1995.

[7] Zinchenko PI. Involuntary memorization. Moscow; MODEK: Voronezh: SAG 1996.
[8] Glozman JM, Potanina AU, Soboleva AE. Neuropsychological assessment in the preschool age. $2^{\text {nd }}$ ed. St. Petersburg: Piter, 2008.

[9] Glozman JM. Quantitative evaluation of neuropsychological assessment data. Moscow: Terevinf 1999.

[10] Elkonin DB. Psychology of play. Moscow: Pedagogica 1978

[11] Glozman JM. Developmental Neuropsychology. London - New York: Psychology Press - Taylor and Francis Group 2013.

Received: January 02, 2015

Revised: February 12, 2015

Accepted: February 20, 2015

(C) Glozman and Konina; Licensee Bentham Open.

This is an open access article licensed under the terms of the Creative Commons Attribution Non-Commercial License (http://creativecommons.org/licenses/bync/3.0/), which permits unrestricted, non-commercial use, distribution and reproduction in any medium, provided the work is properly cited. 